\title{
¿Podemos cambiar nuestros comportamientos ambientales? Un estudio descriptivo con alumnos universitarios
}

\author{
Francisco Javier Perales Palacios. Universidad de Granada \\ David Aguilera Morales. Universidad de Granada
}

Recepción: 19.07.2018| Aceptado: 30.07.2018

Correspondencia a través de ORCID: Javier Perales

iD 0000-0002-6112-2779

Citar: Perales Palacios, FJ. y Aguilera Morales, D. (2018). ¿Podemos cambiar nuestros comportamientos ambientales? Un estudio descriptivo con alumnado universitario. ReiDoCrea, 7, 151-166.

\begin{abstract}
Resumen: Dada la creciente necesidad de cambiar los comportamientos cotidianos de los ciudadanos que inciden negativamente en el medio ambiente, se ha diseñado un cuestionario donde se pregunta a los sujetos sobre algunas de sus conductas que poseen un impacto ambiental. Dicho cuestionario cubre a nuestro juicio un hueco en la literatura educativa, en la medida en que la mayoría de los estudios indagan sobre aspectos cognitivo-afectivos de los comportamientos referidos pero no en las propias acciones. Dicho cuestionario fue aplicado a una muestra de alumnos universitarios españoles que cursaban el grado de Educación Social. Una vez respondidos se debatió en el aula sobre sus respuestas y las alternativas a los comportamientos inadecuados, tras lo cual se les pidió que describieran cuáles eran sus compromisos de cambio y los mecanismos de control que proponían. Se presentan los resultados obtenidos y se analizan de un modo descriptivo, evidenciando un cierto equilibrio entre los comportamientos favorables y desfavorables desde el punto de vista ambiental, aunque los compromisos de mejora y sus controles son respondidos solo parcialmente. Por último se comentan las limitaciones del estudio, las conclusiones más relevantes y las implicaciones educativas del mismo.
\end{abstract}

Palabras clave: Educación Ambiental | Cuestionario

\section{Can we change our environmental behaviors? A descriptive study with university students}

\begin{abstract}
Given the growing need to change the daily behaviors of citizens which have a negative impact on the environment, a questionnaire has been designed to ask subjects about some of their behaviors that have an environmental impact. This questionnaire fills a gap in the educational literature, in that most of the studies investigate cognitive-affective aspects of these behaviors but not the actions themselves. This questionnaire was applied to a sample of Spanish university students studying Social Education. Once answered, their responses and the alternatives to inappropriate behaviors were discussed in the classroom, after which they were asked to describe their commitments to change and the control mechanisms they proposed. The results obtained are presented and a descriptive analysis is carried out, showing a certain balance between favorable and unfavorable behaviors from the environmental point of view, although the commitment to improvement and their control are only partially answered. Finally, the limitations of the study, the most relevant conclusions and the educational implications are discussed.
\end{abstract}

Keywords: Environmental Education | Questionnaires

\section{Introducción}

No creemos preciso insistir en la situación de "emergencia planetaria" a la que la Humanidad está enfrentándose por primera vez en su historia, sin que las alarmas que la comunidad científica viene emitiendo ni otras múltiples evidencias de la gravedad de los problemas ambientales que estamos padeciendo (desastres naturales, desforestación, pérdida de biodiversidad, contaminación...) parezcan servir para convertir la defensa del Medio Ambiente en una prioridad política, social y económica. La metáfora de la "rana hervida" refleja claramente el momento que vivimos: una rana arrojada a un recipiente con agua hirviendo salta y huye del mismo, una rana que se sumerge en agua fría que se va calentando lentamente, se adormece y acaba muriendo de un modo inconsciente. Visto desde una civilización extraterrestre nos 
contemplarían como un colectivo irresponsable que asiste impasible a su autodestrucción.

En lo que sí parece existir consenso es tanto en la complejidad de los problemas ambientales como la de sus soluciones. Estas sin duda pasan precisamente por aunar esfuerzos desde la política, desde el tejido económico-empresarial y desde la modificación de los comportamientos individuales y sociales hacia otros más sostenibles.

A los educadores nos compete desempeñar un relevante papel en el despertar de las conciencias de nuestros estudiantes ante la situación expuesta, lo que implica, entre otras iniciativas, reflexionar sobre nuestros actos cotidianos, mostrar alternativas a los mismos desde un punto de vista de la Sostenibilidad del planeta y comprometernos a una modificación de los mismos hasta convertirlos en hábitos.

Los datos de que disponemos a través de la investigación educativa y sondeos de opinión reflejan que los ciudadanos (especialmente los más jóvenes) suelen estar informados y concienciados sobre los problemas ambientales que nos acucian, pero su grado de compromiso en la solución de los mismos viene a ser débil. De aquí nuestra responsabilidad y retos al respecto.

En este trabajo pretendemos mostrar los resultados de un Cuestionario sobre hábitos cotidianos y su impacto ambiental, así como los compromisos en su mejora por parte de una muestra de estudiantes del grado de Educación Social de la Universidad de Granada que cursan una asignatura optativa relacionada con la Educación Ambiental.

\section{Marco teórico}

Dado que nos vamos a mover en el ámbito de la Educación Ambiental ${ }^{1}$ (EA, en adelante) y por su naturaleza transversal desde el punto de vista académico, las fuentes de las que nos nutriremos van a ser de una procedencia diversa.

Así, el propio concepto de EA viene condicionado por las sucesivas conferencias internacionales que lo han ido delimitando, pero también por las corrientes de pensamiento que cuestionan el modelo de desarrollo socio-económico vigente. Por otro lado, el contexto de las conductas, hábitos y actitudes, en general, viene asimismo a estar influenciado por la psicología social.

Vamos a revisar algunas de las aportaciones que pueden ayudar a entender al problema de investigación que abordamos, esto es, el papel de la EA en el mundo actual y la mejora de los comportamientos pro-ambientales.

Según Novo (2006), la trayectoria de la EA se puede dividir en tres etapas: (1) de 1968 a la década de los 80 (conceptualización); (2) de la década de los 80 a Río 92 (compromiso social y político); y (3) de la década de los 90 a la sociedad global del siglo XXI (la apertura de un nuevo paradigma). Este nuevo paradigma pasa, entre otras cualidades, por vincularse cada vez más estrechamente con los movimientos

\footnotetext{
${ }^{1}$ Las últimas tendencias internacionales vienen a sustituir el término de Educación Ambiental por el de Educación para el Desarrollo Sostenible (EDS) (o también la Educación para la Sostenibilidad). A pesar de las diferencias evidentes (de índole teórica, económica, política...) entre los términos aludidos, por simplificar usaremos prioritariamente la primera (EA) a lo largo del trabajo.
} 
sociales (MS, en adelante) que han venido surgiendo como reacción al modelo capitalista vigente en la mayoría de los países.

En este sentido, Ruiz-Morales (2002), basándose en Ugarte (1996), establece una serie de vinculaciones entre la EA y dichos movimientos:

1. El origen y desarrollo de la EA está ligado a los MS.

2. Ambos están orientados a la resolución de problemas con procesos de reflexión y acción, desde un planteamiento democrático y colectivo.

3. Poseen enfoques interdisciplinarios debido a la complejidad de los conflictos y la realidad socioambiental.

4. La EA se integra en la comunidad, y por tanto en la praxis de los MS, ya que la EA tiene que ser participativa para poder ser concienciadora.

5. Los procesos ambientales, los MS y la EA tienen ritmos semejantes.

6. La EA y los MS son procesos continuos y cambiantes que necesitan de relecturas e interpretaciones, cambios y adaptaciones ante situaciones nuevas.

7. La EA favorece la implicación, compromiso y coherencia de los MS en la realidad socioambiental, a través del análisis de las dimensiones medioambientales para la toma de conciencia y la acción.

8. La EA desde una ética ecológica biocentrista y los MS plantean valores opuestos a los dominantes en el sistema capitalista, para proponer alternativas sustentables, desde la solidaridad, el diálogo, la escucha, el compromiso social, la justicia, la libertad, el reequilibrio sustentable, etc..

Por otro lado, en la Conferencia Mundial sobre la Educación para el Desarrollo Sostenible (2014), se definieron los Objetivos de Desarrollo Sostenible, adoptados por la comunidad mundial, en los que se reconoce la importancia de la educación para la consecución de sus metas en 2030 y estableciéndose el Programa de Acción Mundial, que da seguimiento al Decenio de la Educación para el Desarrollo Sostenible (20052014), y tiene por objeto general ampliar la Educación para el Desarrollo Sostenible y acelerar el progreso hacia el desarrollo sostenible, contribuyendo sustancialmente a la agenda 2030, a través de dos objetivos:

1. Reorientar la educación y el aprendizaje para que todos puedan adquirir conocimientos, habilidades, valores y actitudes que los empoderen y les permitan contribuir a un futuro sostenible.

2. Fortalecer la educación y el aprendizaje en todas las agendas, programas y actividades que promuevan el desarrollo sostenible (Conferencia Mundial sobre la Educación para el Desarrollo Sostenible, 2014).

En definitiva, tanto en el campo de la educación formal como en el de la apertura a los MS, se desprende la urgente necesidad de un cambio en la tendencia autodestructiva de la Humanidad a través del compromiso individual y colectivo en nuestro modo de vida y de pensamiento.

Por otro lado, los comportamientos ambientales pueden ser definidos a partir de su impacto sobre la biosfera, impacto que puede actuar directa (p. ej., la desforestación) o indirectamente ( $p$. ej., la fiscalidad ambiental), así como clasificados de acuerdo a distintas tipologías (Stern, 2000).

Pero al igual que ocurre con la naturaleza de los problemas ambientales, los modelos predictivos de los comportamientos pro-ambientales reflejan su multicausalidad (Stern, 2000) y la complejidad de las relaciones con otras variables y, por tanto, de su consecución (Kollmuss y Agyeman, 2002). En cualquier caso parecen subyacer 
factores demográficos, externos (p. ej., institucionales, económicos, sociales y culturales) e internos (motivación, conocimiento pro-ambiental, conciencia, valores, actitudes, emoción, grado de control, responsabilidades y prioridades) (Kollmuss y Agyeman, 2002; Boyes y Stanisstreet, 2012), a pesar de lo cual estos factores no siempre presentan correlaciones positivas y significativas con tales comportamientos (Castro, Neaman, Vallejo y Elizalde, 2014).Según Gutiérrez-Pérez, Poza y GutiérrezPozo(2015) los factores emocionales se superponen a los racionales y habría de considerarse también la edad de los individuos, especialmente entre la niñez y la adolescencia.

En las investigaciones sobre este tópico se reitera la distancia entre lo que se dice (o se sabe) y lo que se hace a estos efectos, debido sin duda a que algunos de los factores mencionados actúan como freno de los comportamientos deseables. De hecho, la modificación de los comportamientos ambientales a través de la intervención educativa se muestra difícil (Perales, 2010).

En cuanto a las soluciones a los problemas ambientales, los individuos suelen mencionar las científico-tecnológicas, las atribuibles a los organismos internacionales, al aumento de la conciencia ambiental, la disminución del crecimiento económico, el cambio de estilos de vida, de patrones de consumo o de responsabilidades individuales (Tuncer, Sungur, Tekkaya y Ertepinar, 2007).

Dada la incertidumbre señalada y los datos disponibles respecto a la relación entre conocimiento y actitud ambiental (De Chano, 2006), la EA parece reorientarse en los últimos años hacia la promoción de la "competencia para la acción"(Bruun y Schnack, 1997), la cual estaría basada en la reflexión, el pensamiento crítico y la participación, implicando tanto iniciativas individuales como colectivas (Varela, Vega, PérezRodríguez y Álvarez-Lires, 2016), lo que conectaría con la simbiosis EA-MS destacada anteriormente. Estos mismos autores, en su revisión de la literatura sobre dicho tópico, resaltan que la acción debería apoyarse en una toma de decisiones informada tal que permita una transferencia del conocimiento a diferentes contextos y recalibrando las acciones de cada día. Gutiérrez-Pérez et al. (2015) incluso proponen niveles de progresión en la competencia para la sostenibilidad.

No obstante, el salto desde la competencia para la acción a la adquisición de comportamientos ambientales tampoco resulta sencillo. Según Páramo (2017) las formas de abordar la promoción de aquellos se ha basado habitualmente en campañas informativas o de conocimiento ambiental, en técnicas para modificar el comportamiento ambiental o en la inmersión en el medio natural, apoyadas en la investigación en psicología ambiental sobre el cambio actitudinal y la conducta proambiental, suponiendo que los comportamientos sean una manifestación de la actitud, junto con la dimensión cognitiva y la afectiva. Dicho autor propone como alternativa el uso de "reglas ambientales", esto es, "descripciones verbales que establecen relaciones de dependencia entre las ocasiones en que ocurre una conducta, la conducta misma y las consecuencias que esta produce en el ambiente; contingencias que han sido previamente experimentadas o pueden serlo en el futuro" (p. 52). "Las actitudes exploran opiniones, las reglas prescriben lo que hay que hacer" (p. 54). En definitiva, esta perspectiva adopta una postura de índole conductista de cara a la consecución de los objetivos perseguidos.

Acercándonos a la orientación de este trabajo, resulta útil fijarse en el concepto de "intención de conducta", el cual tiene que ver con el compromiso (o no) que pretende adquirir un individuo para llevar a cabo acciones pro-ambientales. Su origen hay que buscarlo en las teorías de "Acción Razonada" y de "Conducta Planificada" (Fishbein y 
Ajzen, 1975; Ajzen y Fishbein, 1980). Así, estos autores sostienen que las actitudes no determinan el comportamiento directamente, sino que influyen en las intenciones de comportamiento que a su vez moldean nuestras acciones. Las intenciones no solo están influenciadas por las actitudes, sino también por las presiones sociales ('normativas'). Por lo tanto, "los determinantes últimos de cualquier comportamiento son las creencias conductuales sobre sus consecuencias y creencias normativas sobre las prescripciones de otros" (Ajzen y Fishbein, 1980, p. 239) (Fig. 1).

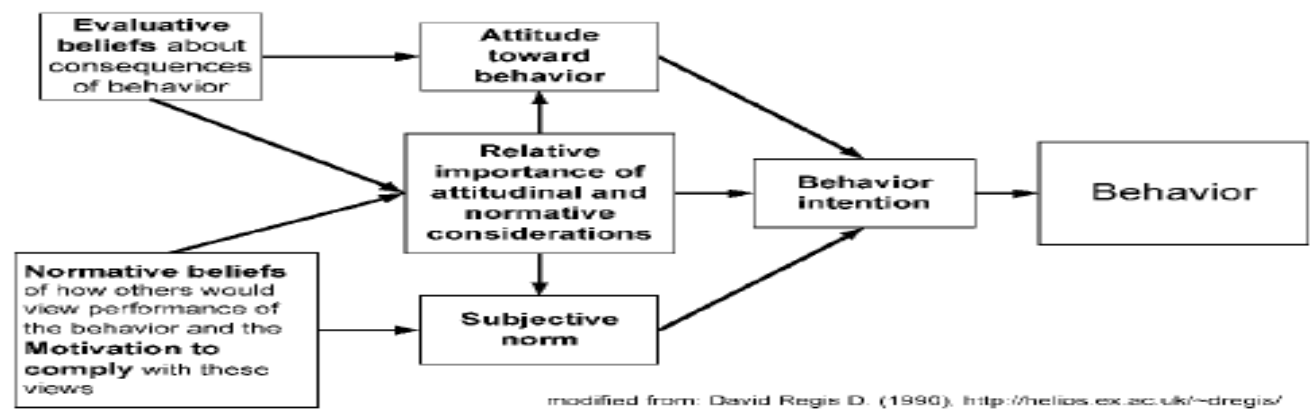

Fig. 1. Representación de la Teoria de acción razonada (Ajzen y Fishbein, 1980).

Dada la complejidad de hallar modelos que expliquen razonablemente las incidencias de los distintos factores psicosociales que influyen en las conductas ambientales, algunos autores recomiendan la medición de aquellos en escenarios reales.

En esta línea, Fujii (2006) obtuvo en una muestra de adultos que la variable facilidad de comportamiento percibido se mostró como un buen determinante de varios comportamientos ambientales (reducciones en el uso de electricidad y gas, basura y uso del automóvil). Otras dos variables analizadas, la preocupación ambiental y la actitud hacia la frugalidad, tuvieron efectos parciales.

Por otro lado, Durán, Alzate, López y Sabucedo (2007), basándose en los trabajos referidos de Fishbein y Ajzen y posteriores, ampliaron el espectro de posibles variables y exploraron la importancia de los aspectos emocionales para comprender y explicar la conducta de reducir la utilización del coche. Así dichos autores encontraron que la ira tuvo una influencia superior a otras variables previstas en la Teoría de Conducta Planificada.

El presente trabajo utiliza un planteamiento pragmático, introduciendo como novedad el utilizar un cuestionario sobre conductas ambientales cotidianas, dado que la mayoría de las investigaciones lo hacen con la vertiente perceptivo-afectiva o con la cognitiva de las mismas (p. ej., Taskin, 2009).

La oportunidad de este trabajo radica en la necesidad de partir de un diagnóstico preciso de los comportamientos ambientales cotidianos de los ciudadanos, lo que debiera servir para la toma de conciencia y debate sobre lo adecuado o inadecuado de los mismos y, en segundo término, constituir la base para forjar la intención de la transformación de los comportamientos menos sostenibles y la subsiguiente adquisición de compromisos al respecto. Ello representa una novedad en el perfil de las investigaciones anteriores sobre este tópico.

\section{Objetivos}

Una vez abordada la reflexión teórica sobre los condicionantes de los comportamientos ambientales, nos planteamos los siguientes objetivos: 
- Implementar un Cuestionario sobre hábitos cotidianos y su impacto ambiental, así como de los compromisos de mejora.

- Aplicarlo a una muestra de estudiantes universitarios con el perfil de educadores sociales.

- Interpretar los resultados en torno a la sostenibilidad de sus acciones y a los compromisos de cambio asumidos.

- Deducir prioridades sobre las que incidir en el ámbito académico universitario.

\section{Metodología}

\section{El contexto}

La experiencia tuvo lugar en el seno de una asignatura optativa del curso $3^{\circ}$ del grado de Educación Social de la Universidad de Granada titulada "Ámbitos, contextos e instrumentos de la Educación Ambiental" (Perales, 2017). Dicha asignatura busca introducir a los estudiantes en la dimensión social de la EA mediante un programa que se estructura básicamente en dos partes, asumidas respectivamente por sendos profesores de las áreas de conocimiento de Didáctica de las Ciencias Experimentales (el autor) y del área de Métodos de Investigación y Diagnóstico en Educación. Dichas partes contemplan los siguientes contenidos:

1. Introducción a la EA y a la Sostenibilidad. Problemáticas ambientales actuales. Estrategias metodológicas en la EA. Intervención en la Educación formal.

2. TIC y alfabetizacion ambiental. Ecologismo y Movimientos proambientales. Gestión e Intervención educativa en contextos no formales e informales. Evaluación de programas, centros, materiales, recursos y cambios personales. Competencias profesionales y perfil del educador social en programas de EA.

En concreto, el Cuestionario se les pasó a los estudiantes en la parte 1, una vez vistos los contenidos de Introducción a la EA y a la Sostenibilidad, y Problemáticas ambientales actuales. Así, se planteó tras la revisión del concepto de actitud ambiental y su relación con otros conceptos axiológicos como valores, normas, creencias, hábitos o emociones, intentando dar respuesta al lema: "Un reto difícil pero ineludible, el cambio comportamental".

\section{La muestra}

El número de alumnos matriculados en la asignatura fue de 66 (12 hombres y 54 mujeres) pero la asistencia y la cumplimentación del Cuestionario fue menor. De hecho, 53 lo hicieron con aquel y 46 con la cuestión posterior.

\section{El instrumento}

El diseñar el Cuestionario utilizado en esta investigación ha venido dado por la ausencia de precedentes en la línea que deseábamos (solo hemos encontrado una referencia no publicada al "Inventario de conducta ambiental responsable"de N. J. Smith-Sebasto, 1995).

La "filosofía" del Cuestionario sobre hábitos cotidianos y su impacto ambiental era la de que los alumnos de la asignatura pudieran recorrer mentalmente las acciones cotidianas que llevaran a cabo desde que se levantan hasta que se acuestan y reflexionar sobre su impacto ambiental. Se trata de las conductas ambientales descritas por Stern (2000) como "ambientalismo de la esfera privada". Con ello se pretendía testear el perfil de los comportamientos ambientales de la muestra 
participante. Dicho Cuestionario fue ajustado durante los cursos previos a través de la observación por parte del autor de tales acciones y de las correcciones surgidas a través de la interacción con los propios alumnos.

El Cuestionario constaba de un total de 68 ítems organizados en torno a actividades principales y, en algunos casos, actividades secundarias (Tabla IV). Los ítems estaban planteados en forma interrogativa con tres opciones: sí, no, a veces; tras lo cual se disponía de otras columnas en las que se les preguntaba si podrían mejorar y se les proporcionaban otras tres posibilidades: sí, no, no sé. Las cuestiones que se plantean a los estudiantes van dirigidas a detectar si sus conductas habituales son favorables 0 no para la sostenibilidad del ambiente. En concreto, 51 ítems recogían conductas consideradas como ambientalmente favorables (FA) y los 17 restantes como desfavorables (DA).

A los alumnos se les pidió, como actividad propia de la asignatura, que rellenaran el Cuestionario fuera del horario lectivo y lo subieran a la plataforma docente que utiliza la universidad. En la clase siguiente se debatieron las implicaciones ambientales de las conductas planteadas en el Cuestionario y, en su caso, las alternativas a las mismas.

Para la clase posterior se les solicitó que añadieran al cuestionario ya resuelto las respuestas a la cuestión siguiente: "De las distintas preguntas formuladas en el Cuestionario, ¿cuáles nos comprometemos a mejorar y cómo vamos a controlar su cumplimiento?". Para ello se añadieron dos nuevas columnas, una para que especificaran el compromiso de mejora y otra para el cómo lo controlarían. Tanto las tres últimas columnas del Cuestionario como esta pregunta adicional buscaban sondear la intención de conducta pro-ambiental de los estudiantes. Al igual que antes, hubieron de subir sus respuestas a la plataforma de uso docente.

\section{Análisis de datos}

Dado el carácter prospectivo y eminentemente cuantitativo del Cuestionario, su análisis será meramente descriptivo. Ello se hará mediante la determinación de porcentajes de respuesta a los ítems, en función de su idoneidad ambiental, agrupándolos posteriormente en torno a las actividades principales. Seguidamente se llevará a cabo otro análisis similar para la cuestión posterior al cuestionario y un análisis por categorías de las respuestas al "Cómo pensaban controlar el cumplimiento del compromiso adquirido".

\section{Resultados}

\section{Análisis de las respuestas globales}

Los porcentajes promedio totales de los 68 ítems, separados en FA o DA y tipo de respuesta se muestran en la Tabla I.

\begin{tabular}{l} 
Tabla I. Porcentajes promedio de respuesta de la muestra a los ítems del cuestionario clasificados como FA o DA y \\
tipos de respuesta a los mismos \\
\hline
\end{tabular}


Un primer análisis de estos datos globales nos permite inferir que, en general, las conductas FA prevalecen de un modo claro sobre las DA (57\% frente a un $30 \%$ de respuestas afirmativas, respectivamente), aunque si se considera la opción de "a veces" y se suma a la de "no", tendríamos que para las conductas FA, éstas supondrían un $57 \%$ y la suma de las otras dos opciones un $42 \%$. En el caso de las DA, las respuestas afirmativas más las de "a veces" representan un $64 \%$ frente a un $36 \%$ que declaran no llevarlas a cabo. Esto nos proporciona un panorama en el que los comportamientos FA y DA presentan un cierto equilibrio.

En cuanto a las posibilidades de mejora de los comportamientos, las respuestas afirmativas son claramente superiores a las negativas o a las "no sé". Esto supone que, en el caso de las respuestas FA, las primeras aparezcan en un $53 \%$, frente a un $48 \%$ de la suma de las otras dos; de todas formas hay que tener en cuenta que las respuestas negativas suelen darse porque los sujetos consideran que no pueden mejorar dado que lo están haciendo correctamente; en cambio los "no sé" pueden significar una manifestación de sus dudas respecto a si hay alternativas mejores a las conductas que desarrollan. Para los comportamientos DA, las respuestas afirmativas suponen un $61 \%$, en comparación con el $43 \%$ de la suma de las otras dos alternativas; ello es coherente con la toma de conciencia por parte de los sujetos de que están frente a respuestas poco adecuadas en cuanto a su incidencia en el ambiente; asimismo las respuestas negativas, como anteriormente referíamos, se producen porque los sujetos piensan que no incurren en conductas inadecuadas.

\section{Análisis de las respuestas por actividad principal}

Vamos a desglosar a continuación (Tabla II) los porcentajes promedio en función de las actividades principales del cuestionario que se mostraron en la Tabla I.

\begin{tabular}{|c|c|c|c|c|c|c|}
\hline \multicolumn{7}{|c|}{ Ambientalmente favorables $(\mathrm{N}=51)$} \\
\hline Actividad principal & Sí & No & A veces & \multicolumn{3}{|c|}{ ¿Podría mejorar? } \\
\hline & & & & Sí & No & No sé \\
\hline Higiene & $44 \%$ & $40 \%$ & $15 \%$ & $45 \%$ & $32 \%$ & $17 \%$ \\
\hline Movilidad & $49 \%$ & $28 \%$ & $23 \%$ & $59 \%$ & $21 \%$ & $20 \%$ \\
\hline Alimentación & $54 \%$ & $26 \%$ & $21 \%$ & $49 \%$ & $25 \%$ & $26 \%$ \\
\hline Colada & $59 \%$ & $20 \%$ & $20 \%$ & $44 \%$ & $36 \%$ & $20 \%$ \\
\hline Limpieza del hogar (1 ítem) & $29 \%$ & $46 \%$ & $25 \%$ & $63 \%$ & $12 \%$ & $25 \%$ \\
\hline Climatización y ventilación & $68 \%$ & $19 \%$ & $13 \%$ & $43 \%$ & $36 \%$ & $21 \%$ \\
\hline Consumo & $68 \%$ & $9 \%$ & $23 \%$ & $61 \%$ & $23 \%$ & $15 \%$ \\
\hline Facturas & $46 \%$ & $34 \%$ & $20 \%$ & $62 \%$ & $14 \%$ & $24 \%$ \\
\hline \multicolumn{7}{|c|}{ Ambientalmente desfavorables ( $\mathrm{N}=17$ ) } \\
\hline Actividad principal & Sí & No & A veces & \multicolumn{3}{|c|}{ ¿Podría mejorar? } \\
\hline & & & & Sí & No & No sé \\
\hline Higiene & $27 \%$ & $38 \%$ & $35 \%$ & $62 \%$ & $27 \%$ & $11 \%$ \\
\hline Alimentación & $24 \%$ & $37 \%$ & $44 \%$ & $64 \%$ & $19 \%$ & $16 \%$ \\
\hline Colada & $57 \%$ & $28 \%$ & $14 \%$ & $37 \%$ & $19 \%$ & $43 \%$ \\
\hline Limpieza del hogar & $32 \%$ & $41 \%$ & $26 \%$ & $54 \%$ & $23 \%$ & $22 \%$ \\
\hline $\begin{array}{l}\text { Climatización y ventilación } \\
\text { (1 ítem) }\end{array}$ & $29 \%$ & $37 \%$ & $33 \%$ & $82 \%$ & $4 \%$ & $14 \%$ \\
\hline Consumo & $42 \%$ & $19 \%$ & $38 \%$ & $78 \%$ & $10 \%$ & $11 \%$ \\
\hline Ocio(1 ítem) & $10 \%$ & $63 \%$ & $27 \%$ & $42 \%$ & $38 \%$ & $20 \%$ \\
\hline
\end{tabular}


A la vista de la Tabla II se deduce que las conductas FA (respuestas "sí", por un lado, y la suma de los "no" y "a veces", por el otro) que más destacan es en los apartados de climatización-ventilación y consumo, y donde menos, en la limpieza del hogar (aunque en este último caso solo se incluía un ítem). En cuanto a las posibilidades de mejora, además de la ya mencionada de limpieza del hogar, aparecen destacadas las facturas, el consumo y la movilidad; las dudas al respecto ("no sé") sufren pocas variaciones, oscilando en torno al $20 \%$.

Siguiendo las pautas anteriores, si nos fijamos ahora en los comportamientos DA y sumamos los "sí" y los "a veces", sobresalen los referentes al consumo, seguido de la colada, y el que menos, el ítem relativo al ocio, no existiendo grandes diferencias en el resto. Por lo que respecta al margen de mejora, destacan los relativos a la climatización-ventilación y consumo, y los menos representados los de la colada y el ocio. Las incertidumbres inherentes a las respuestas "no sé" no presentan grandes diferencias, excepto las respuestas referentes a la colada.

\section{Análisis de las respuestas por actividad principal, secundaria e ítem}

Ahora vamos a centrarnos en los ítems con respuestas extremas agrupados en las actividades principales y secundarias. Para ello representamos en la Tabla III los ítems que han recibido unos porcentajes de respuesta de sí o no con valores comprendidos entre el $0-10 \%$ y el $90-100 \%$. Hemos añadido también la columna de "a veces" para poder matizar mejor el alcance de esas respuestas dicotómicas.

\begin{tabular}{|c|c|c|c|c|c|}
\hline Actividad principal & $\begin{array}{l}\text { Actividad } \\
\text { Secundaria }\end{array}$ & Ítem & Sí & No & $\begin{array}{c}A \\
\text { veces }\end{array}$ \\
\hline \multirow[t]{5}{*}{ Higiene } & Ducha & ¿Aprovechas con un cubo el agua fría hasta que sale la caliente? & $5 \%$ & & $17 \%$ \\
\hline & \multirow[t]{4}{*}{ Lavabo } & ¿Cierras el grifo mientras te lavas los dientes? & $95 \%$ & & $1 \%$ \\
\hline & & ¿Empleas jabón de pastilla? & $9 \%$ & & $16 \%$ \\
\hline & & $\begin{array}{l}\text { ¿Descongelas los alimentos pasándolos previamente al } \\
\text { compartimento del refrigerador? }\end{array}$ & $7 \%$ & & $38 \%$ \\
\hline & & ¿Cierras el grifo mientras enjabonas la vajilla? & & $5 \%$ & $18 \%$ \\
\hline \multirow[t]{5}{*}{ Colada } & & ¿Empleas casi siempre agua fría? & & $5 \%$ & $32 \%$ \\
\hline & & ¿Echas sólo la cantidad de detergente necesaria? & & $10 \%$ & $29 \%$ \\
\hline & & ¿Pones suavizante?* & $96 \%$ & $0 \%$ & $4 \%$ \\
\hline & & ¿Secas la ropa al aire libre? & & $4 \%$ & $15 \%$ \\
\hline & & ¿Echas mucho detergente en el agua?* & $6 \%$ & & $31 \%$ \\
\hline \multirow{12}{*}{$\begin{array}{l}\text { Climatización y } \\
\text { ventilación }\end{array}$} & & ¿Ventilas la casa a diario? & & $6 \%$ & $17 \%$ \\
\hline & & ¿Aprovechas el calor o el frío "naturales" para acondicionarla? & & $6 \%$ & $39 \%$ \\
\hline & & ¿Dispones de cortinas? & & $10 \%$ & $0 \%$ \\
\hline & & ¿Te vistes de acuerdo a la época del año? & $100 \%$ & $0 \%$ & $0 \%$ \\
\hline & & ¿Prefieres la luz natural a la artificial? & $92 \%$ & $2 \%$ & $6 \%$ \\
\hline & & ¿Apagas las luces cuando no son necesarias? & & $0 \%$ & $29 \%$ \\
\hline & & ¿La coses cuando es necesario? & & $4 \%$ & $17 \%$ \\
\hline & & ¿Intentas reparar los objetos que se nos rompen? & & $2 \%$ & $23 \%$ \\
\hline & & $\begin{array}{l}\text { ¿Te preocupa el coste social, en materias primas, en su } \\
\text { transformación y transporte que tiene el hecho de comprar productos } \\
\text { manufacturados? }\end{array}$ & & $6 \%$ & $22 \%$ \\
\hline & & $\begin{array}{l}\text { ¿Piensas en la gran cantidad de personas que disponen de muchos } \\
\text { menos recursos que nosotros? }\end{array}$ & $92 \%$ & $0 \%$ & $8 \%$ \\
\hline & & ¿Reutilizas las bolsas que te dan en el supermercado? & $94 \%$ & $0 \%$ & $6 \%$ \\
\hline & & ¿Has elegido la compañía eléctrica con menor impacto ambiental? & $6 \%$ & & $18 \%$ \\
\hline Ocio & & $\begin{array}{l}\text { ¿Necesitas beber para pasártelo bien o te sientes mal si no haces lo } \\
\text { que la mayoría?* }\end{array}$ & $10 \%$ & & $17 \%$ \\
\hline
\end{tabular}


Analizando globalmente los porcentajes de respuestas afirmativas y negativas, en todos los casos se corresponden a ítems FA, excepto tres: “¿Pones suavizante?”; ¿Echas mucho detergente en el agua?; ¿Necesitas beber para pasártelo bien o te sientes mal si no haces lo que la mayoría? En el primer ítem, vemos que el uso de suavizante es generalizado, lo que demuestra que desconocen los riesgos que para la salud representan algunos de los componentes y que prima el tacto y el olor de la ropa; es algo similar a lo que ocurre con los ambientadores sintéticos. Aunque con porcentajes bajos, los efectos de los otros dos ítems son claros, el primero sobre la calidad de las aguas residuales y el segundo sobre la salud y la generación de residuos.

Respecto al resto de los ítems, algunas de las respuestas presentan unos porcentajes que se pueden interpretar como poco FA, tal es el caso de: ¿Aprovechas con un cubo el agua fría hasta que sale la caliente?; ¿Empleas jabón de pastilla?; ¿Descongelas los alimentos pasándolos previamente al compartimento del refrigerador? o ¿Has elegido la compañía eléctrica con menor impacto ambiental? En el primero, una medida tan sencilla como disponer de un cubo en la bañera, nos permite ahorrar agua utilizándola en el WC. El segundo supone un ahorro en el uso de jabón (la duración es bastante mayor que el del jabón líquido) más el propio envase de este último. Para el tercer ítem parece claro que no lo realizan frecuentemente, seguramente porque falta planificación del menú y porque disponen de microondas con función de descongelación; ello provoca un malgasto energético, tanto por no aprovechar la baja temperatura del alimento previamente congelado para ahorrar energía en el refrigerador como por el propio gasto del microondas. El cuarto ítem implica la libre elección de una compañía eléctrica y el conocimiento de las fuentes energéticas de las que se abastecen (renovables o no renovables); esta información aparece en las facturas que emiten las compañías eléctricas pero hay que tener en cuenta que son escasos los estudiantes universitarios que están emancipados y, por tanto, disponen de esa capacidad autónoma de elección.

La lectura de la última columna de la Tabla III nos permite visualizar que, en algunos casos, aunque sea de forma intermitente, los comportamientos que inicialmente pudieran parecer como FA, pueden tener una componente de DA.

\section{Análisis de las respuestas a la cuestión posterior a la cumplimentación del Cuestionario}

Como ya avanzamos, tras haber respondido los estudiantes al mencionado Cuestionario, se debatió en el aula cada uno de los ítems, estableciéndose un intercambio de experiencias entre ellos y actuando el profesor como mediador y orientador cuando los propios estudiantes no conocían alternativas a las conductas ambientalmente negativas. Una vez finalizada la sesión de clase, como actividad semanal se les propuso que respondieran y subieran a la plataforma docente: "De las distintas preguntas formuladas en el Cuestionario, ¿cuáles nos comprometemos a mejorar y cómo vamos a controlar su cumplimiento?".

En la Figura 1 se representan gráficamente las frecuencias globales de los compromisos adquiridos por los estudiantes y de las medidas de control que proponen. Por otro lado, sus respuestas fueron agrupadas por ítem y frecuencia (Tabla IV). 


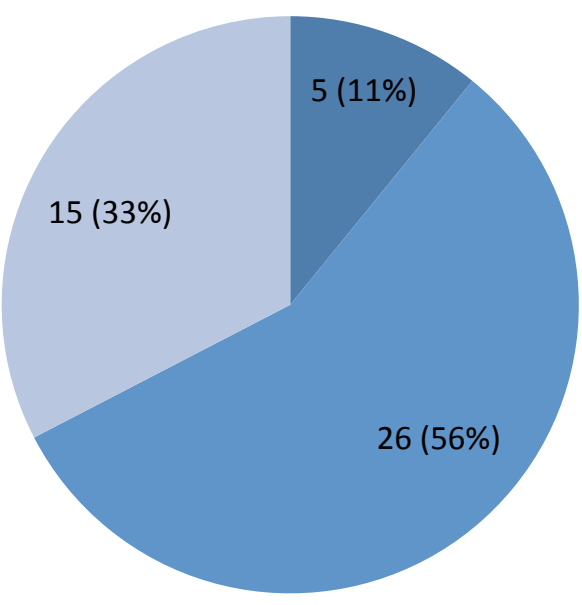

Medidas de control para cada uno de los compromisos - Medidas de control para algunos de los compromisos Ninguna medida de control

Figura 1. Análisis de frecuencias y porcentajes para las medidas de control establecidas por los estudiantes.

\begin{tabular}{|c|c|c|c|c|c|}
\hline $\begin{array}{l}\text { Actividad } \\
\text { principal }\end{array}$ & $\begin{array}{l}\text { Activi } \\
\text { Secur }\end{array}$ & $\begin{array}{l}\text { idad } \\
\text { Indaria }\end{array}$ & Ítem & \begin{tabular}{|l|}
$\begin{array}{l}\text { Frecuencia } \\
(\%)^{1}\end{array}$ \\
\end{tabular} & Medidas de control (frecuencia) $^{2}$ \\
\hline \multirow[t]{9}{*}{ Higiene } & \multirow{4}{*}{\multicolumn{2}{|c|}{ Ducha }} & $\begin{array}{l}\text { ¿Aprovechas con un cubo el agua fría } \\
\text { hasta que sale la caliente? }\end{array}$ & $27(59 \%)$ & $\begin{array}{l}\text { Usar el agua para limpieza del hogar, regar las } \\
\text { plantas o para el WC (18) }\end{array}$ \\
\hline & & & $\begin{array}{l}\text { ¿Sueles ducharte con agua a una } \\
\text { temperatura elevada?*}\end{array}$ & $9(20 \%)$ & Ajustar el termostato del calentador eléctrico (4) \\
\hline & & & $\begin{array}{l}\text { ¿Cortas la ducha mientras te } \\
\text { enjabonas? }\end{array}$ & $16(35 \%)$ & \\
\hline & & & ¿Usas más jabón del necesario?* & $10(22 \%)$ & $\begin{array}{l}\text { Colocar cartel informativo sobre la dosis de } \\
\text { jabón a emplear (2) }\end{array}$ \\
\hline & \multirow{3}{*}{\multicolumn{2}{|c|}{ Lavabo }} & $\begin{array}{l}\text { ¿Cierras el grifo mientras te lavas los } \\
\text { dientes? }\end{array}$ & $7(15 \%)$ & \\
\hline & & & ¿Empleas jabón de pastilla? & $14(30 \%)$ & \\
\hline & & & $\begin{array}{l}\text { ¿Enciendes más luces de las } \\
\text { necesarias?* }\end{array}$ & $8(17 \%)$ & \\
\hline & \multirow{2}{*}{\multicolumn{2}{|c|}{$W C$}} & $\begin{array}{l}\text { ¿Descargas completa o parcialmente la } \\
\text { cisterna en función de la necesidad? }\end{array}$ & $9(20 \%)$ & $\begin{array}{l}\text { Introducir un objeto pesado (botella con arena, } \\
\text { ladrillo...) para reducir la capacidad de la } \\
\text { cisterna (3) }\end{array}$ \\
\hline & & & ¿Utilizas el WC como papelera?* & $11(24 \%)$ & \\
\hline \multicolumn{4}{|c|}{$\begin{array}{l}\text { Media del número de personas que se comprometen a mejorar en esta } \\
\text { categoría }\end{array}$} & \multicolumn{2}{|l|}{$12(26 \%)$} \\
\hline \multicolumn{4}{|c|}{ Total de compromisos } & \multicolumn{2}{|l|}{111} \\
\hline \multirow{6}{*}{\multicolumn{2}{|c|}{ Movilidad }} & $\begin{array}{l}\text { Acceso } \\
\text { vivienda }\end{array}$ & $\begin{array}{l}\text { Si vives en un piso, ¿subes o bajas a } \\
\text { pie o en el ascensor?* }\end{array}$ & $7(15 \%)$ & \\
\hline & & \begin{tabular}{|l|} 
Medio de \\
transporte
\end{tabular} & ¿Utilizas uno público? & $8(17 \%)$ & $\begin{array}{l}\text { Contabilidad del dinero mensual en combustible } \\
\text { (2) }\end{array}$ \\
\hline & & & ¿Compartes vehículo? & $4(9 \%)$ & \\
\hline & & & $\begin{array}{l}\text { ¿Practicas técnicas de conducción para } \\
\text { gastar menos combustible? }\end{array}$ & $7(15 \%)$ & \\
\hline & & & $\begin{array}{l}\text { ¿Podrías hacer el trayecto total o parcial } \\
\text { caminando? }\end{array}$ & $9(20 \%)$ & \\
\hline & & & ¿Te has planteado utilizar la bicicleta? & $12(26 \%)$ & \\
\hline \multicolumn{4}{|c|}{$\begin{array}{l}\text { Media del número de personas que se comprometen a mejorar en esta } \\
\text { categoría }\end{array}$} & \multicolumn{2}{|l|}{$8(17 \%)$} \\
\hline \multicolumn{4}{|c|}{ Total de compromisos } & 47 & \\
\hline \multirow[t]{2}{*}{ Alimentación } & \multirow[t]{2}{*}{ Dieta } & \multirow{2}{*}{\multicolumn{2}{|c|}{ ¿Usas preferentemente productos frescos? }} & $9(20 \%)$ & $\begin{array}{l}\text { Elaborar un esquema con los productos de } \\
\text { temporada para cada mes del año y colocarlo en } \\
\text { la nevera (2) }\end{array}$ \\
\hline & & & & $9(20 \%)$ & Comprar productos a granel (2) \\
\hline
\end{tabular}




\begin{tabular}{|c|c|c|c|c|}
\hline & & $\begin{array}{l}\text { ¿Te interesas por el origen geográfico de los } \\
\text { alimentos? }\end{array}$ & $16(35 \%)$ & $\begin{array}{l}\text { Comprar los alimentos en comercios pequeños y } \\
\text { locales (5) } \\
\text { Mirar las etiquetas de los productos (5) } \\
\text { Destinar más tiempo a la compra (2) }\end{array}$ \\
\hline & & $\begin{array}{l}\text { ¿Has comprado alguna vez productos } \\
\text { ecológicos? }\end{array}$ & $7(15 \%)$ & \\
\hline & & ¿Tiras la comida sobrante?* & $5(11 \%)$ & \\
\hline & & $\begin{array}{l}\text { ¿Utilizas habitualmente para beber agua } \\
\text { embotellada?*}\end{array}$ & $3(7 \%)$ & \\
\hline & Frigorífico & $\begin{array}{l}\text { ¿Crees que almacenas correctamente los } \\
\text { alimentos en el frigorífico? }\end{array}$ & $9(20 \%)$ & \\
\hline & & ¿Los tienes demasiado tiempo almacenados? & $7(15 \%)$ & $\begin{array}{l}\text { Comprar menos cantidad de alimentos (3) } \\
\text { Controlar las fechas de caducidad (2) }\end{array}$ \\
\hline & & $\begin{array}{l}\text { ¿Adaptas la temperatura de aquel al volumen } \\
\text { de carga del mismo? }\end{array}$ & $8(17 \%)$ & \\
\hline & & ¿Es un electrodoméstico clase $A ?$ & $3(7 \%)$ & \\
\hline & & $\begin{array}{l}\text { ¿Descongelas los alimentos pasándolos } \\
\text { previamente al compartimento del refrigerador? }\end{array}$ & $4(9 \%)$ & \\
\hline & Otros & ¿Utilizas la olla a presión para cocinar? & $2(4 \%)$ & \\
\hline & & $\begin{array}{l}\text { ¿Ajustas la temperatura de la cocina cuando } \\
\text { los alimentos hierven? }\end{array}$ & $2(4 \%)$ & \\
\hline & & $\begin{array}{l}\text { ¿Aprovechas la "inercia térmica" en el horno o } \\
\text { en la cocina de vitrocerámica? }\end{array}$ & $5(11 \%)$ & \\
\hline & & ¿Utilizas mucho jabón para fregar la vajilla?* & $5(11 \%)$ & \\
\hline & & ¿Cierras el grifo mientras la enjabonas? & $3(7 \%)$ & \\
\hline & & $\begin{array}{l}\text { ¿Si tienes lavavajillas, lo llenas del todo antes } \\
\text { de conectarlo? }\end{array}$ & 0 & \\
\hline & & ¿Pones un programa económico? & $4(9 \%)$ & \\
\hline \multirow{2}{*}{\multicolumn{3}{|c|}{$\begin{array}{l}\text { Media del número de } \\
\text { categoría }\end{array}$}} & \multicolumn{2}{|l|}{$6(13 \%)$} \\
\hline & & & 101 & \\
\hline \multirow[t]{6}{*}{ Colada } & & ¿Empleas casi siempre agua fría? & $3(7 \%)$ & \\
\hline & & \begin{tabular}{|l}
$\begin{array}{l}\text { ¿Echas sólo la cantidad de detergente } \\
\text { necesaria? }\end{array}$ \\
\end{tabular} & $4(9 \%)$ & \\
\hline & & ¿Pones suavizante?* & $7(15 \%)$ & \\
\hline & & ¿Sueles utilizar lejía?* & $6(13 \%)$ & \\
\hline & & ¿Secas la ropa al aire libre? & $3(7 \%)$ & \\
\hline & & ¿Aprovechas el sol para blanquear la ropa? & $6(13 \%)$ & \\
\hline \multicolumn{3}{|c|}{$\begin{array}{l}\text { Media del número de personas que se comprometen a mejorar en esta } \\
\text { categoría }\end{array}$} & \multicolumn{2}{|l|}{$5(11 \%)$} \\
\hline \multicolumn{3}{|c|}{ Total de compromisos } & \multicolumn{2}{|l|}{29} \\
\hline \multirow[t]{3}{*}{\begin{tabular}{|l|} 
Limpieza del \\
hogar
\end{tabular}} & & $\begin{array}{l}\text { ¿Usas productos alternativos como el vinagre o } \\
\text { el limón? }\end{array}$ & $7(15 \%)$ & \\
\hline & & ¿Utilizas lejía?* & $4(9 \%)$ & Utilizar productos naturales (2) \\
\hline & & ¿Echas mucho detergente en el agua?* & $3(7 \%)$ & \\
\hline \multicolumn{3}{|c|}{$\begin{array}{l}\text { Media del número de personas que se comprometen a mejorar en esta } \\
\text { categoría }\end{array}$} & \multicolumn{2}{|l|}{$5(11 \%)$} \\
\hline \multicolumn{3}{|c|}{ Total de compromisos } & \multicolumn{2}{|l|}{14} \\
\hline \multirow{11}{*}{\begin{tabular}{l|} 
Climatiza-ción y \\
ventila-ción
\end{tabular}} & & ¿Ventilas la casa a diario? & $2(4 \%)$ & \\
\hline & & $\begin{array}{l}\text { ¿Aprovechas el calor o el frío "naturales" para } \\
\text { acondicionarla? }\end{array}$ & $5(11 \%)$ & \\
\hline & & ¿Tienes aisladas las ventanas y las puertas? & $2(4 \%)$ & \\
\hline & & ¿Dispones de cortinas? & $2(4 \%)$ & \\
\hline & & ¿Y toldos? & $1(2 \%)$ & \\
\hline & & ¿Te vistes de acuerdo a la época del año? & $1(2 \%)$ & \\
\hline & & $\begin{array}{l}\text { ¿Conoces el coste económico y ambiental del } \\
\text { tipo de calefacción-refrigeración que usas? }\end{array}$ & $6(13 \%)$ & \\
\hline & & ¿Prefieres la luz natural a la artificial? & $1(2 \%)$ & \\
\hline & & ¿Apagas las luces cuando no son necesarias? & 0 & \\
\hline & & $\begin{array}{l}\text { ¿Tienes lámparas de bajo consumo o LED al } \\
\text { menos en las habitaciones en las que estás } \\
\text { más tiempo? }\end{array}$ & $5(11 \%)$ & \\
\hline & & ¿Dejas los electrodomésticos en "stand by"?* & $7(15 \%)$ & \\
\hline
\end{tabular}




\begin{tabular}{|c|c|c|}
\hline \multirow{2}{*}{$\begin{array}{l}\begin{array}{l}\text { Media del número de personas que se comprometen a mejorar en esta } \\
\text { categoría }\end{array} \\
\text { Total de compromisos }\end{array}$} & \multirow{2}{*}{\multicolumn{2}{|c|}{\begin{tabular}{|l|}
$3(7 \%)$ \\
32
\end{tabular}}} \\
\hline & & \\
\hline \multirow[t]{11}{*}{ Consumo } & $5(11 \%)$ & $\begin{array}{l}\text { Evitar aquellas marcas o empresas que usan un } \\
\text { embalaje excesivo (2) }\end{array}$ \\
\hline & $8(17 \%)$ & \\
\hline & $5(11 \%)$ & \\
\hline & $3(7 \%)$ & \\
\hline & 0 & \\
\hline & $3(7 \%)$ & \\
\hline & $3(7 \%)$ & \\
\hline & $1(2 \%)$ & \\
\hline & $16(35 \%)$ & $\begin{array}{l}\text { Colocar tres papeleras, una para cada tipo de } \\
\text { residuo, en el mismo lugar }(6)\end{array}$ \\
\hline & $2(4 \%)$ & \\
\hline & $2(4 \%)$ & \\
\hline $\begin{array}{l}\text { Media del número de personas que se comprometen a mejorar en esta } \\
\text { categoría }\end{array}$ & \\
\hline Total de compromisos & \multicolumn{2}{|l|}{41} \\
\hline \multirow[t]{2}{*}{ Facturas } & $3(7 \%)$ & \\
\hline & $3(7 \%)$ & \\
\hline $\begin{array}{l}\text { Media del número de personas que se comprometen a mejorar en esta } \\
\text { categoría }\end{array}$ & \multicolumn{2}{|l|}{$3(7 \%)$} \\
\hline Total de compromisos & \multicolumn{2}{|l|}{6} \\
\hline \multirow[t]{2}{*}{ Ocio } & 0 & \\
\hline & $2(4 \%)$ & \\
\hline $\begin{array}{l}\text { Media del número de personas que se comprometen a mejorar en esta } \\
\text { categoría }\end{array}$ & \multicolumn{2}{|l|}{$1(2 \%)$} \\
\hline Total de compromisos & \multicolumn{2}{|l|}{2} \\
\hline
\end{tabular}

Como podemos comprobar de la Figura 1 y de la Tabla IV, la proporción de estudiantes que plantean compromisos de mejora de sus conductas ambientales es estimable, aunque con evidentes diferencias según la actividad de que se trate, alcanzando el máximo en higiene y el mínimo en ocio. Llama la atención a este respecto, que el consumo (o consumismo) acapare solo un $9 \%$ de estudiantes que adquieren compromisos de mejora, dado que se considera como el principal agente causal del deterioro ambiental que padecemos.

Por otro lado, cuando se trata de establecer mecanismos de control para esos compromisos, el nivel de respuesta desciende taxativamente, quedándose en solo un $11 \%$ los sujetos que proponen mecanismos para todos sus compromisos. Dichos mecanismos, salvo excepciones, presentan pocas afinidades. Destacaremos por frecuencia decreciente los siguientes (Tabla IV):

- Usar el agua para limpieza del hogar, regar las plantas o para el WC

- Colocar tres papeleras, una para cada tipo de residuo, en el mismo lugar

- Comprar los alimentos en comercios pequeños y locales

- Mirar las etiquetas de los productos 


\section{Discusión}

Comenzaremos recordando los objetivos que nos marcamos al inicio de este trabajo:

- Implementar un Cuestionario sobre hábitos cotidianos y su impacto ambiental, así como de los compromisos de mejora.

- Aplicarlo a una muestra de estudiantes universitarios con el perfil de educadores sociales.

- Interpretar los resultados en torno a la sostenibilidad de sus acciones y a los compromisos de cambio asumidos.

- Deducir prioridades sobre las que incidir en el ámbito académico universitario.

Estimamos que los tres primeros se han cumplido y en este apartado abordaremos el cuarto.

En relación a las limitaciones de la investigación, dado su carácter meramente exploratorio, no cabe hablar de validez externa. Hemos de tener en cuenta que se trata de una muestra de un tamaño limitado y con un perfil definido, como es de los estudiantes del grado universitario de Educación Social. Es previsible que con otro perfil de estudiantes, los resultados hubieran diferido dado que este grado suele abastecerse de personas con una motivación hacia la resolución de los problemas sociales (Iglesias y Meira, 2007; Perales, 2017). Asimismo, el uso de un Cuestionario no garantiza que las respuestas se correspondan fielmente con las conductas cotidianas, aunque el carácter de la actividad no implicaba ninguna repercusión académica más allá de tener que responderlo. Como alternativa solo queda un seguimiento de aquellos comportamientos a través de indicadores objetivos como el consumo eléctrico (Frazer y Leslie, 2014).

Por otro lado, el análisis de datos efectuado podría haberse complementado con una diferenciación por sexo para dilucidar si existían diferencias significativas entre ambos colectivos y por tipo de actividad. Asimismo, podríamos haber cuantificado las respuestas individuales en cuanto a su carácter FA o DA, lo que nos hubiera permitido un análisis estadístico más profundo.

En cuanto a los resultados obtenidos en la aplicación del Cuestionario y la actividad posterior sobre compromisos y mecanismos de control, se puede decir que globalmente los estudiantes de la muestra poseen unos comportamientos ambientales a medio camino entre los favorables y los desfavorables, lo que permite un amplio rango de mejora. Ello es reconocido mayoritariamente cuando se pregunta a los participantes en el estudio sobre si podrían mejorar tales comportamientos, aunque persisten dudas al respecto en torno a un $20 \%$ de la muestra.

De las actividades principales analizadas, destacan favorablemente las de climatización y ventilación, y en sentido contrario la limpieza del hogar. Por otra parte $y$, en sentido contrario, nos encontramos a las actividades de consumo y colada.

Cuando se hace una lectura más detallada de los porcentajes extremos por ítem emergen algunas conductas inapropiadas, como el uso masivo de suavizante para el lavado de la ropa o la descongelación inadecuada de alimentos, entre otras.

Si nos detenemos ahora en los compromisos de mejora y el modo de control que sugieren los estudiantes, los participantes tienen un nivel de concienciación ambiental elevado y su predisposición para revertir aquellas conductas DA es satisfactoria. Sin embargo, solo una minoría de los participantes ha sido capaz de diseñar estrategias 
que controlen cada una de las conductas que desean mejorar, mientras que algo más de la mitad ha proporcionado medidas de control parciales, cubriendo solamente algunos de los compromisos realizados. Finalmente, un tercio de la muestra parece incapaz de establecer medidas que pudieran controlar sus conductas y compromisos medioambientales, hecho que podría estar condicionado por falta de conocimiento práctico sobre dicha temática (tal y como afirman varios de los estudiantes en sus manuscritos). Esta afirmación se corrobora por la dispersión de las propuestas de mecanismos de control que sugieren.

Pasaremos finalmente a hablar de las implicaciones educativas de este trabajo. Pensamos en primer lugar que una actividad de aula, como la llevada a cabo en nuestro caso, en la que se debatan los resultados del Cuestionario realizado previamente y se discutan posibles alternativas a las conductas DA, constituye una herramienta útil para la ambientalización del currículo de diversas materias educativas. No obstante, como señalaron Frazer y Leslie (2014), el tiempo de retroalimentación y los objetivos planteados, así como la cadencia de estos, pueden modificar algunos comportamientos ambientales (en su caso, el uso de la electricidad en los hogares). Es decir, sería preciso un seguimiento periódico del cumplimiento de los compromisos y un apoyo explícito con información directa y comprensible pero también con mensajes emocionalmente estimulantes.

Por último, este trabajo nos ha permitido refinar más el Cuestionario empleado, elaborando una versión final depurada que pondremos a disposición de la comunidad educativa.

Los educadores, por su proyección social, se han de convertir en faros que guíen, a través de su propio compromiso y ejemplo diario, el comportamiento ambiental de sus educandos, pero para ello debieran haber interiorizado previamente ese cambio de estilo de vida y valores que conlleva ser ambientalmente responsable.

\section{Referencias}

Ajzen, I. y Fishbein, M. (1980) Understanding Attitudes and Predicting Social Behavior. Englewood Cliffs, NJ: Prentice Hall.

Barazarte, R., Neaman, A., Vallejo, F. y García-Elizalde, P. (2014). El conocimiento ambiental y el comportamientoproambiental de los estudiantes de la Enseñanza media en la Región de Valparaíso (Chile). Revista de Educación, 364, 12-34.

Boyes, E. y Stanisstreet, M. (2012). Environmental Education for Behaviour Change: Which actions should be targeted? International Journal of Science Education, 34(10), 1591-1614.

Bruun, BJ. y Schnack, K. (1997) The Action Competence Approach in Environmental Education, Environmental Education Research, 3(2), 163-178.

Castro, RB. Neaman, A., Vallejo, F. y Elizalde, P. (2014). El conocimiento ambiental y el comportamiento proambiental de los estudiantes de la Enseñanza media, en la Región de Valparaíso (Chile). Revista de Educación, 364, 12-34.

Conferencia Mundial sobre la Educación para el Desarrollo Sostenible (2014). Aichi Nagoya, Japón. Recuperado de https://es.unesco.org/gap

DeChano, LM. (2006) A multi-country examination of the relationship between environmental knowledge and attitudes.
International Research in Geographical and Environmental Education, 15(1), 15-28.

Durán, M., Alzate, M., López, W. y Sabucedo, JM. (2007). Emociones y comportamiento pro-ambiental. Revista Latinoamericana de Psicología, 39(2), 287-296.

Fishbein, M. y Ajzen, I. (1975). Belief, Attitude, Intention, and Behavior: An introduction to theory and research. Reading, MA: Addison-Wesley.

Frazer, P. y Leslie, J. (2014). Feedback and goal-setting interventions to reduce electricity use in the real world. Behavior and Social Issues, 23, 20-34

Fujii, S. (2006). Environmental concern, attitude toward frugality, and ease of behavior as determinants of pro-environmental behavior intentions. Journal of Environmental Psychology, 26, 262-268.

Gutiérrez-Pérez, J., Poza, MF. y Gutiérrez-Pozo, M. (2015). Progresión-disrupción en el desarrollo de actitudes ambientales. Alambique Didáctica de las Ciencias Experimentales, 79, 45-52.

Iglesias, L. y Meira, P. (2007). De la Educación Ambiental a la Educación Social o viceversa. Educación Social, 35, 13-27. 
Kollmuss, A. y Agyeman, J. (2002). Mind the gap: Why do people act environmentally and what are the barriers to pro-environmental behavior? Environmental EducationResearch, 8(3), 240-260.

Novo, M. (2006). El desarrollo sostenible: su dimensión ambiental y educativa. Madrid: Pearson Educación.

Páramo, P. (2017). Reglas proambientales: una alternativa para disminuir la brecha entre el decir-hacer en la educación ambiental. Suma Psicológica ,2(4), 42-58.

Perales-Palacios, FJ. (2010). Cambios en la percepción ambiental de los futuros maestros de Educación Primaria. Comunicación al II Congreso Internacional de Didácticas (Gerona, 3-6 Febrero).

Perales-Palacios, FJ. (2017). Educación ambiental y educación social: el punto de vista de los estudiantes. ReiDoCrea, 6, 1 - 15.

Ruiz-Morales, J. (2002). Movimientos sociales y educación ambiental. Hacia la construcción de una pedagogía transformadora. Revista Kikiriki. Cooperación educativa, 64, 13-20.

Stern, PC. (2000). Toward a Coherent Theory of Environmentally Significant Behavior. Journal of Social Issues, 56(3), 407-424.

Taskin, O. (2009). The Environmental Attitudes of Turkish Senior High School Students in the Context of Postmaterialism and the New Environmental Paradigm. International Journal of Science Education, 31 (4), 481-502.

Tuncer, G., Sungur, S., Tekkaya, C. y Ertepinar, H. (2007). A Comparative Study on Pre-Service Teachers' and Elementary Students' Attitudes towards the Environment. International Research in Geographical and Environmental Education, 16(2), 188-198.

Varela-Losada, M., Vega-Marcote, P., Pérez-Rodríguez, U. y Álvarez-Liresa, M. (2016). Going to action? A literature review on educational proposals informal Environmental Education. Environmental Education Research, 22(3), 390-421. 